\title{
The electroweak phase transition at $m_{H} \simeq 80 \mathrm{GeV}$ from $L_{t}=2$ lattices*
}

Ferenc Csikor ${ }^{\mathrm{a}}$, Zoltan Fodor ${ }^{\mathrm{b} \dagger}$ and Jochen Heitger ${ }^{\mathrm{c}}$

${ }^{a}$ Institute for Theoretical Physics, Eötvös University, H-1088 Budapest, Hungary

b KEK Theory Group, Tsukuba-shi, Ibaraki 305, Japan

c Institut für Theoretische Physik I, Universität Münster, D-48149 Münster, Germany

We study the finite-temperature electroweak phase transition by numerical simulations of the four-dimensional $\mathrm{SU}(2)$-Higgs model on anisotropic lattices with temporal extension $L_{t}=2$. The physically interesting parameter region of Higgs masses near $80 \mathrm{GeV}$ is reached, and recent results on some thermodynamic quantities are presented.

\section{Introduction}

Though the scenario of electroweak baryogenesis at a sufficiently strong first order phase transition [1] within the SM seems to be ruled out [2,3], it appears important to quantify its nature and strength at more realistic Higgs masses $m_{H} \simeq 80 \mathrm{GeV}$, and to compare with effective $3 D$-theories claiming an endpoint of the transition line at $m_{H}^{\text {(crit) }} \lesssim 80 \mathrm{GeV}$, beyond which the EWPT turns into an analytic crossover [2]. Hence we made numerical simulations of the anisotropic $\mathrm{SU}(2)-$ Higgs model, since for weaker transitions at larger $m_{H}$ one expects the typical excitations $m \ll T$ to require isotropic lattices exceeding most accessible computer resources.

In the following we will focus on interface tension and latent heat from $T>0$ simulations at $L_{t}=2 \ll L_{\mathrm{x}, \mathrm{y}} \ll L_{\mathrm{z}}$. These, involving a sequence of heatbath and overrelaxation algorithms, were done at HLRZ, Jülich (CRAY-T90), and DESYIfH, Zeuthen (APE-Quadrics), Germany.

\section{Anisotropic SU(2)-Higgs model}

The lattice action of the four-dimensional $\mathrm{SU}(2)$-Higgs model on anisotropic lattices reads

$S[U, \varphi]=\sum_{x}\left\{\sum_{i=s, t} \beta_{i} \sum_{\mathrm{p}_{i}}\left(1-\frac{1}{2} \operatorname{Tr} U_{\mathrm{p}_{i}, x}\right)\right.$

\footnotetext{
${ }^{*}$ Poster presented by J. H. at LATTICE 97, Edinburgh. ${ }^{\dagger}$ On leave from Institute for Theoretical Physics, Eötvös University, H-1088 Budapest, Hungary.
}

$$
\begin{aligned}
& -\kappa_{s} \sum_{\mu=1}^{3} \operatorname{Tr}\left(\varphi_{x+\hat{\mu}}^{+} U_{x, \mu} \varphi_{x}\right)-\kappa_{t} \operatorname{Tr}\left(\varphi_{x+\hat{4}}^{+} U_{x, 4} \varphi_{x}\right) \\
& \left.+\frac{1}{2} \operatorname{Tr}\left(\varphi_{x}^{+} \varphi_{x}\right)+\lambda\left[\frac{1}{2} \operatorname{Tr}\left(\varphi_{x}^{+} \varphi_{x}\right)-1\right]^{2}\right\}
\end{aligned}
$$

in terms of gauge links $U_{x, \mu} \in \mathrm{SU}(2)$, space- and

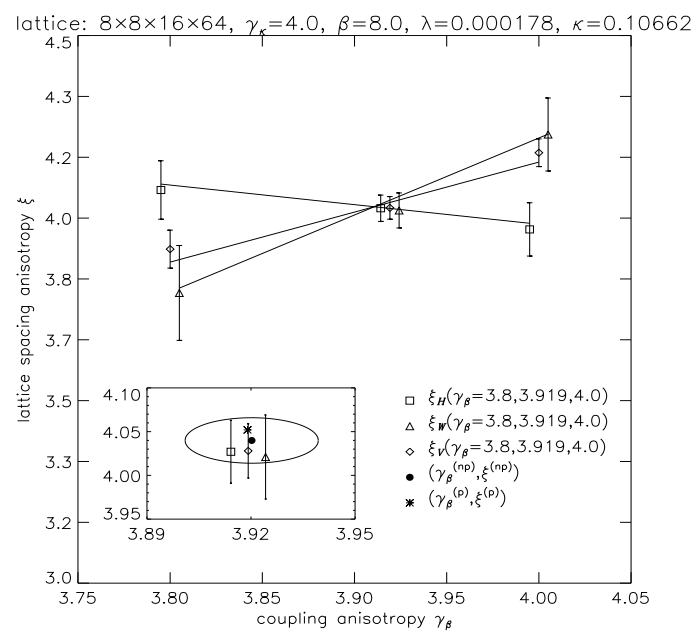

Figure 1. $\xi$-evaluation at $m_{H}=72(5) \mathrm{GeV}$ and $g_{R}^{2}=0.577(15)$, whose equal abscissas are displaced. $\xi_{i}\left(\gamma_{\beta}\right)$, as $s$ - to $t$-like $m_{i}$-ratios in the Higgs and vector channels $(i=H, W)$ and from a suitable mapping of static potentials $(i=V)$, are interpolated to coincide in errors. The error ellipse of the matching point encloses the numerical estimates and the perturbative one [4].

timelike plaquettes $U_{\mathrm{p}_{s}, x}$ and $U_{\mathrm{p}_{t}, x}$, site variables 
$\varphi_{x}=\rho_{x} \alpha_{x}, \rho_{x}>0, \alpha_{x} \in \mathrm{SU}(2)$, and the lattice spacing and coupling anisotropy parameters $\xi \equiv a_{s} / a_{t}, \gamma_{\beta} \equiv \sqrt{\beta_{t} / \beta_{s}}$, and $\gamma_{\kappa} \equiv \sqrt{\kappa_{t} / \kappa_{s}}$ with $\beta^{2}=\beta_{s} \beta_{t}$ and $\kappa^{2}=\kappa_{s} \kappa_{t}$. The general strategy of $T>0$ studies in $D=4$ is to fix $T_{c}=1 / a_{t} L_{t}$ at a given temporal extension $L_{t}$, to determine the critical hopping parameter $\kappa_{c}$, and to calculate in this phase transition point the physical, non-perturbatively renormalized parameters $R_{H W} \equiv m_{H} / m_{W}\left(m_{W}^{\text {(phys) }}=80 \mathrm{GeV}\right)$ and $g_{R}^{2}$ in simulations on $T=0$ lattices [3]. Then the continuum limit is realized as approach to the scaling region via $L_{t} \rightarrow \infty$ along lines of constant physics.

In [4] we confirmed the one-loop corrections $\gamma_{\beta}^{(\mathrm{p})}=3.919$ and $\xi^{(\mathrm{p})}=4.052$ to the tree-level anisotropies $\gamma_{\beta}=\gamma_{\kappa} \equiv \xi \equiv 4$ non-perturbatively by demanding space-time symmetry restoration (rotational invariance) with correlation lengths in physical units being equal in both directions, see figure 1. This opens the way to analyze the EWPT for $m_{H} \gtrsim 80 \mathrm{GeV}$ within the $4 D$-model in a systematic and fully controllable way.

\section{Thermodynamic quantities and results}

In view of the large lattices to be used, the interface tension $\sigma$ has been determined by employing the two-coupling method [5] in $\kappa$, which in previous investigations of the $\mathrm{SU}(2)$-Higgs model [6] turned out to be quite robust and, at the same time, most economic among the other methods at disposal 77. After enforcing an interface pair perpendicular to the $\mathrm{z}$-direction by dividing the lattice volume in symmetric and Higgs phases with $\left(\kappa_{1}<\kappa_{c}: \mathrm{z} \leq L_{\mathrm{z}} / 2, \kappa_{2}>\kappa_{c}: \mathrm{z}>L_{\mathrm{z}} / 2\right)$, the related additional free energy $\Delta F$ yields for $\Delta \kappa \equiv \kappa_{2}-\kappa_{1} \ll 1$ the estimator [3.,6]

$a_{s}^{2} a_{t} \sigma=\frac{1}{2} \lim _{\Delta \kappa \rightarrow 0}\left\{\Delta \kappa \cdot L_{\mathrm{z}} \cdot\left[L_{\varphi}^{(1)}-L_{\varphi}^{(2)}\right]\right\}$.

$L_{\varphi}^{(i)}=L_{\varphi}^{(i)}\left(\kappa_{1}, \kappa_{2}\right)$ denotes the expectation value of the $\varphi$-link operator $L_{\varphi ; x \mu} \equiv \frac{1}{2} \operatorname{Tr}\left(\varphi_{x+\hat{\mu}}^{+} U_{x, \mu} \varphi_{x}\right)$ in the respective phases, and, since $\Delta F \simeq \mathcal{O}(\Delta \kappa)$, the $(N+2)$-parametric Laurent ansätze

$$
L_{\varphi}^{(i)}=-\frac{c_{i}}{\kappa_{i}-\kappa_{c}}+\sum_{j=0}^{N} \gamma_{i}^{(j)}\left(\kappa_{i}-\kappa_{c}\right)^{j}+\cdots
$$

give $\hat{\sigma} / T_{c}^{3}=L_{t}^{3} L_{z}\left(c_{1}+c_{2}\right) / \xi^{2}$. As exemplarily displayed in figures 2 and 3 , we performed such fits to sets of $2-\kappa$ data at $L_{t}=2$ with simulation parameters corresponding to $m_{H}=78(4)$ $\mathrm{GeV}$ pole mass and $g_{R}^{2}=0.539(16)$. For the

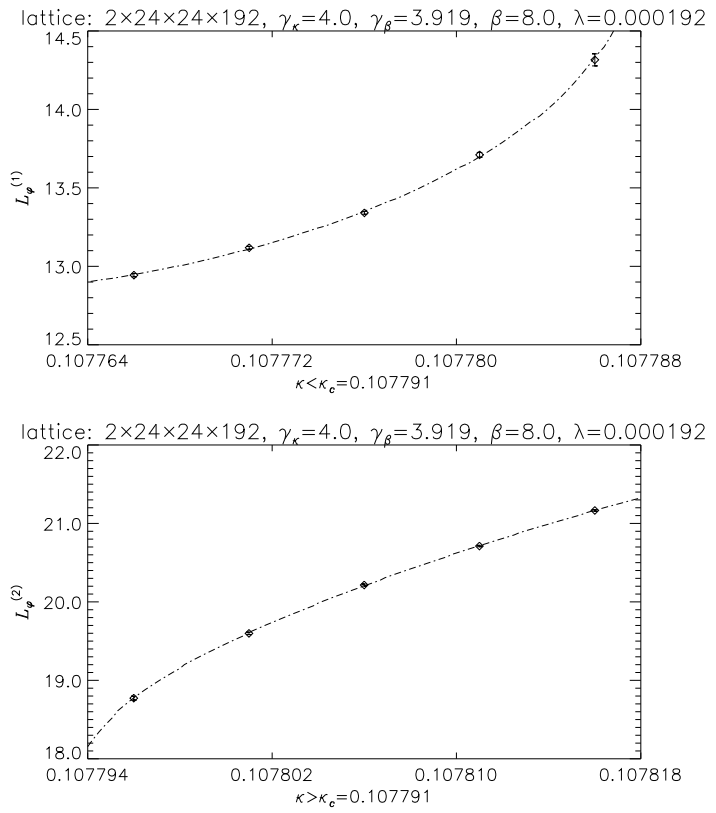

Figure 2. Four-parameter $\chi^{2}$-fit of $L_{\varphi}^{(i)}, i=1,2$.

best fit we found $\hat{\sigma} / T_{c}^{3}=0.0006(3)$ on a lattice of size $2 \times 24^{2} \times 192$ with $\chi^{2} /$ dof $\simeq 1$, in complete agreement with some data from a larger spatial volume. When inspecting various fits along the available $\kappa$-intervals with a reasonable number of fit parameters $\gamma_{i}^{(j)}$ in eq. (3), the combined number quoted in table 1 covers the total spread of all reliable fit results, whose individual errors include the statistical error from a bootstrap analysis and the significant uncertainty in $\kappa_{c}$ [6].

From quadratic fits of the discontinuities of the order parameters showing up in the thermal cycles of figure 1 we also extracted the jump in the Higgs field vacuum expectation value, here as $\Delta v / T_{c}=L_{t} \xi^{-1} \sqrt{2 \kappa \Delta\left\langle\rho^{2}\right\rangle}$, and in $L_{t}^{4} \xi^{-3} \frac{\partial \kappa}{\partial \tau} \Delta\left\langle L_{\varphi}\right\rangle, \tau \equiv-\ln \left(a_{t} m_{W}\right)$, which is the dominating contribution to the latent heat if defined as the energy density difference $\Delta \epsilon / T_{c}^{4}$ [3]. Their numerical outcomes at identical parameters are collected in table 1 as well. 


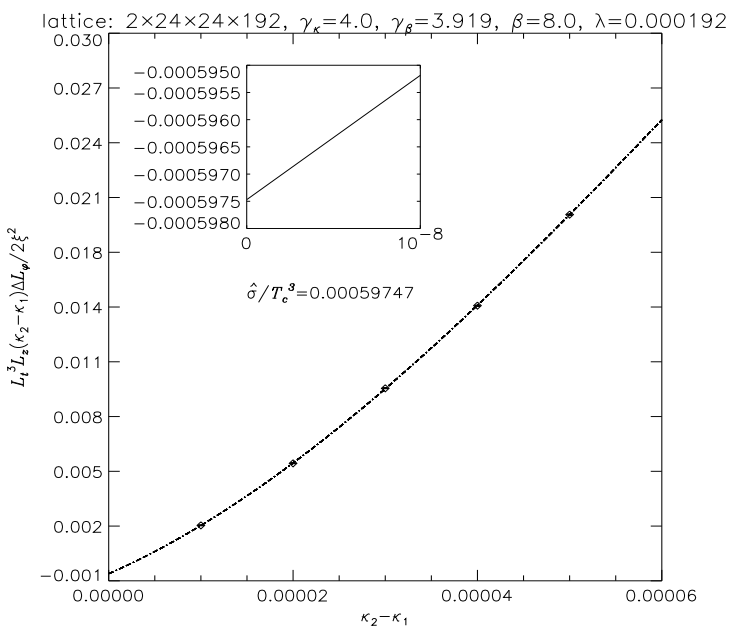

Figure 3. As in figure 目 but for $\Delta L_{\varphi} \equiv L_{\varphi}^{(2)}-$ $L_{\varphi}^{(1)}$.

\begin{tabular}{ccccc}
\hline$L_{t}$ & $T_{c} / m_{H}$ & $10^{4} \hat{\sigma} / T_{c}^{3}$ & $\Delta v / T_{c}$ & $10^{4} \Delta \epsilon / T_{c}^{4}$ \\
\hline \hline 2 & $1.86(2)$ & $6(4)$ & $0.37(16)$ & $33(27)$ \\
3 & $1.8(2)$ & - & - & - \\
\hline
\end{tabular}

Table 1. Lattice results at $L_{t}=2$ and, preliminarily, at $L_{t}=3$. The transition points lie at $\kappa_{c}=0.107791(3)$ and $\kappa_{c}=0.10703(3)$.

\section{Discussion and outlook}

$\hat{\sigma} / T_{c}^{3}$ and $\Delta \epsilon / T_{c}^{4}$ for $m_{H} \simeq 80 \mathrm{GeV}$ are substantially smaller than perturbatively $\left(\sigma / T_{c}^{3} \simeq\right.$ 0.002 [8]). They are even consistent with a no first order phase transition scenario approximately on the $1-\sigma$ level. The fact that this result deviates from those of the $3 D$-investigations [2] should be clarified in future. However, a temporal lattice extension of $L_{t}=2$ may be still too far from continuum physics, and at least the knowledge of the behaviour at $L_{t}=3$ seems necessary to draw a final conclusion.

\section{REFERENCES}

1. D. A. Kirzhnitz, JETP Lett. $\underline{15}$ (1972) 529; D. A. Kirzhnitz, A. D. Linde, Phys. Lett. B72 (1972) 471; Ann. Phys. 101 (1976) 195; V. A. Kuzmin, V. A. Rubakov, M. Shaposhnikov, Phys. Lett. B155 (1985) 36; M. Shaposhnikov, Nucl. Phys. B287 (1987) 757.

2. K. Kajantie et al., Phys. Rev. Lett. 77 (1996)
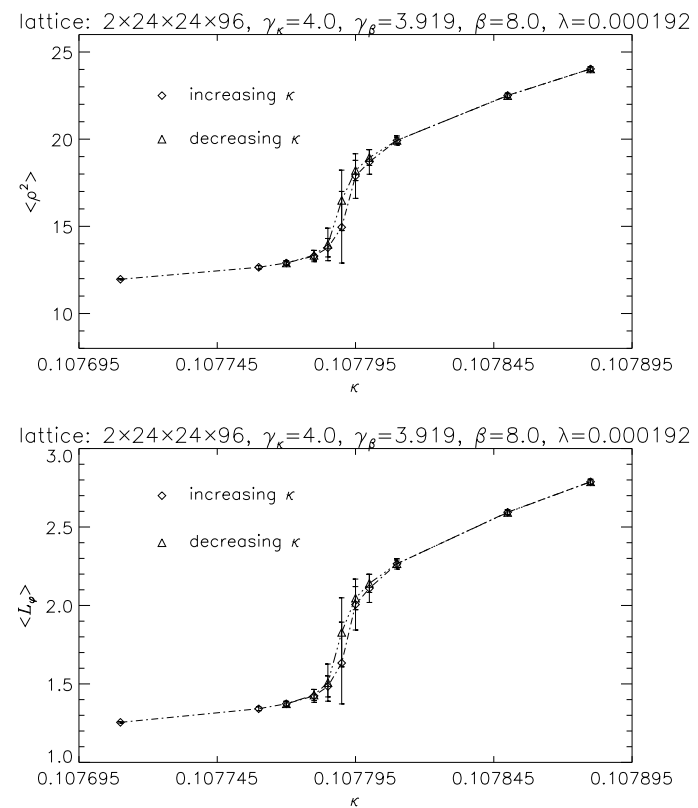

Figure 4. Hystereses of the operators $\rho^{2}$ and $L_{\varphi}$ around the critical $\kappa$-region at $L_{t}=2$.

2887; Nucl. Phys. B493 (1997) 413; F. Karsch et al., Nucl. Phys. B53 (Proc. Suppl.) (1997) 623; M. Gürtler, E.-M. Ilgenfritz, A. Schiller, UL-NTZ-10-97 (hep-lat/9704013), 1997 and these proceedings.

3. Z. Fodor et al., Nucl. Phys. B439 (1995) 147;

F. Csikor et al., Nucl. Phys. B474 (1996) 421.

4. F. Csikor, Z. Fodor, Phys. Lett. B380 (1996) 113; F. Csikor, Z. Fodor, J. Heitger, in preparation.

5. J. Potvin, C. Rebbi, Phys. Rev. Lett. $\underline{62}$ (1989) 3062; S. Huang et al., Phys. Rev. D42 (1990) 2864; Phys. Rev. D43 (1991) 2056 [E].

6. F. Csikor, Z. Fodor, J. Hein, J. Heitger, Phys. Lett. B357 (1995) 156; J. Hein, J. Heitger, Phys. Lett. B385 (1996) 242.

7. K. Binder, Z. Phys. B43 (1981) 119; Phys. Rev. A25 (1982) 1699; K. Jansen et al., Nucl. Phys. B322 (1989) 698; M. Gürtler, E.-M. Ilgenfritz, A. Schiller, UL-NTZ-08-97 heplat/9702020), 1997 and these proceedings.

8. Z. Fodor, A. Hebecker, Nucl. Phys. B432 (1994) 127; W. Buchmüller, Z. Fodor, A. Hebecker, Phys. Lett. B331 (1994) 131; Nucl. Phys. B447 (1995) 317. 